\title{
Gilt das Stereotyp der polnischen Wirtschaft immer noch? Zu den Veränderungen in Polen aus imagologischer Perspektive
}

\author{
Does the stereotype of the Polish economy \\ still exist? About changes in Poland from \\ the imagological perspective
}

\begin{abstract}
The main focus the paper investigates concerns Polish-German relations from the perspective of imagology. The author of the article concentrates on the stereotype of the polish economy and compares it with the imagological standpoint by analyzing two chosen publications: „Viva Polonia. Als deutscher Gastarbeiter in Polen" by Steffen Möller and „Polen" by Thomas Urban. The above-mentioned analysis should lead to an answer for the following question: can the way of perceiving of the Polish economy be modified by means of well-known authors?
\end{abstract}

KEYWORDS: German-Polish relations, stereotypes, Polish economy as a stereotype, imagology.

\section{EINLEITUNG}

Das 21. Jahrhundert wird in Polen mit den unterschiedlichen Veränderungen assoziiert. Zahlreiche Lebensbereiche werden mit den verschiedenartigen Entwicklungen assoziiert - dazu gehören beispielsweise Wirtschaft, Politik und Kultur. Ein Schwerpunkt dieses Artikels besteht darin, die gegenwärtigen Bilder der polnischen Gesellschaft zusammenzustellen und einer kritischen Untersuchung zu unterziehen. Nach Orłowski (2004: 14) wird das jahrelang existierende und stark geprägte Stereotyp der polni- 
schen Wirtschaft mit der Rückständigkeit, mangelhaften Entwicklung und ökonomischen Innovationsunfähigkeit assoziiert. An dieser Stelle soll bemerkt werden, dass der Ausdruck "polnische Wirtschaft” in seinen Diskursen als multidimensional bezeichnet wird. Der oben erwähnte Begriff wird im Deutschen nicht mehr erstrangig als Stereotyp, sondern vielmehr in direkten fachlichen Bezeichnungen gebraucht. Andererseits gilt das Stereotyp der polnischen Wirtschaft aus der Perspektive der Imagologie und interkulturellen Kommunikation als eine Quelle der verschiedenartigen Inhalte. Der vorliegende Artikel versteht sich in diesem Zusammenhang als Versuch, die bisher bekannte Einstellung zu der polnischen Wirtschaft zu diskutieren und aus der Sichtweise der Imagologie zu betrachten, d.h. die überall bekannten Texte zum Thema Polenbilder einer Analyse zu unterziehen, um die Frage zu beantworten, ob die in den Veröffentlichungen dargestellten Bilder imstande sind, das Stereotyp der polnischen Wirtschaft zu modifizieren. Erwähnenswert bleibt die Tatsache, dass dieser Aufsatz versucht, das Stereotyp und die Fremdwahrnehmungsbilder Polens miteinander in unterschiedliche Beziehungen zu setzen. Die ausgewählten Publikationen werden in der deutschen und polnischen Gesellschaft als populär klassifiziert. Aus diesem Grund können sie die öffentliche Meinung beeinflussen. Bei dem Versuch, das Phänomen der polnischen Wirtschaft zu skizzieren, muss es gerade um die Frage gehen, ob dieses Stereotyp im Hinblick auf die in den ausgewählten Texten dargestellte positive Perspektive Modifikationen unterliegen kann.

\section{DAS STEREOTYP DER POLNISCHEN WIRTSCHAFT}

Die Verfasserin dieses Artikels versucht, die meinungsbildende Funktion des Stereotyps der polnischen Wirtschaft anhand von Texten zu untersuchen. Zuerst Betrachtung verdient jedoch die Klärung des Begriffs. Nach Auderska, Łempicka, Skorupka (1968: 777) oder Chlebda (1998: 31) werden Stereotype in den unterschiedlichen Fachrichtungen auf verschiedene Art und Weise definiert. Sie werden z.B. aus soziologischer oder psychologischer Perspektive oder aus der Sichtweise der Geschichte und Literatur in Erwägung gezogen. In der alltäglichen Umgangssprache werden Stereotype und Vorurteile oft fälschlicherweise als Synonyme betrachtet - dies wird von Auerheimer als "fehlerhafte und starre Verallgemeinerung" (Auerheimer 1990: 1433) verstanden. Einen ähnlichen Standpunkt vertritt auch Hansen: "Der Begriff des Vorurteils ist mit dem des Stereotyps verwandt" (Hansen 2000: 322). Der Ansatz von Schneider ähnelt den Feststellungen von Auerheimer und Hansen: "There is no doubt that our prejudices, however derived, can sometimes drive our stereotypes and make them 
resistant to change" (Schneider 1996: 440). Der Begriff des Stereotyps wird zum ersten Mal im Jahr 1922 in der Veröffentlichung des US-amerikanischen Schriftstellers, Journalisten und Medienkritikers Walter Lippmanns unter dem Titel „Die öffentliche Meinung” („Public Opinion”) geklärt. Die Bezeichnung der Stereotype als "pictures in our heads" von Lippmann revolutionierte die bisherige Betrachtungsweise der gegenseitigen Bilder:

For the most part we do not first see, and then define, we define first and then see. In the great blooming, buzzing confusion of the outer world we pick out what our culture has already defined for us, and we tend to perceive that which we have picked out in the form stereotyped for us by our culture (Lippmann 1922/2008: 37).

Der erwähnte Begriff des Stereotyps wurde auch später und wird immer noch aus unterschiedlichen Perspektiven von verschiedenen Wissenschaftlern analysiert. Stereotype werden laut Orłowski (2004: 14f) als generalisierende Meinungen eines Individuums oder einer Gruppe angesehen:

Generell wird das Stereotyp als eine Bewusstheitsform definiert, die der Erfahrung grundsätzlich fern steht. Daher wird auch der individuellen Wahrnehmung der Umwelt eine geringere Bedeutung zugemessen als der gesellschaftlichen Überlieferung, der Tradition. Umso stärker wird der Charakter der „subjektiven Selbstverständlichkeit" herausgehoben (Orłowski 2004: 14f).

Auf diese Art und Weise entstehen nach Lippmann bestimmte Vorstellungen einer Kultur, die der Realität eines Kulturraums auf keinen Fall entsprechen: „Ein Stereotyp kann die Wirklichkeit verfehlen oder in seiner Verallgemeinerung zu weit gehen. Beides kann die Erkenntnis des Fremden sicherlich blockieren, doch diese Blockade ist durch Überprüfung abzubrechen" (Lippmann 1922/2008: 37).

Adler, Gundersen vertreten die Meinung, dass Stereotype modifiziert werden können. Der Prozess der Modifizierung soll jedoch mit einer bestimmten Regel konnotiert werden: „,continuing observation and experience with the actual people and situations" (Adler, Gundersen 2008: 77). Andererseits ist nach Adler und Gundersen das Modifizieren des Stereotyps mit verschiedenartigen Schwierigkeiten und Missverständnissen verbunden: „Because we believe stereotypes reflect reality, subconsciously held stereotypes are difficult to modify or discard even after we acquire real information about a person" (Adler, Gundersen 2008: 77). Ähnliche Ansichten vertreten auch andere Wissenschaftler, wie z.B. die folgenden: Hogg, Vaughan (2010: 194), Stangor (2000: 10) oder Fiske, Operario (2004: 16). 
Die Autorin dieses Artikels konzentriert sich auf einem bestimmten Stereotyp - auf die Betrachtungsweise der polnischen Wirtschaft im deutschpolnischen Kontext. Mit diesem Aspekt beschäftigen sich unterschiedliche Forscher wie z.B.: Papiór (1997: 95), Fiedor (1997: 92) oder Golec (2000: 306). Erwähnenswert bleibt die Tatsache, dass die Mehrheit der Veröffentlichungen zum Thema polnische Wirtschaft von dem polnischen Germanisten und ehemaligen Mitarbeiter der Adam-Mickiewicz-Universität Posen, Hubert Orłowski, publiziert wurde. Wie bereits angedeutet, wird das Stereotyp der polnischen Wirtschaft mit der Rückständigkeit und der mangelhaften Entwicklung assoziiert ${ }^{1}$. Dazu zahlen auch laut Orłowski solche Aspekte wie: „niedrige Motive und Instinkte (Trunk) statt patriotischer Gefühle, Inkompetenz, Chaos und Ineffizienz" 2 . Die weiteren Untersuchungen des Begriffs führen zu den folgenden Schlussfolgerungen:

Auf die kürzeste Formel gebracht bedeutet die Wendung soviel wie chaotisches, also letztendlich ineffizient organisiertes Handeln. Sie prägte das Polenbild der frühen und späten Neuzeit bis tief ins 20. Jahrhundert In seiner monopolistischen Deutungsfunktion stand das Stereotyp in wechselseitiger Beziehung zum bürgerlichen Bewusstsein. Insbesondere prägte es negativ-positiv polarisierende Alltagsvorstellungen von eigenen Sekundärtugenden wie Ordnung, Fleiß, Sparsamkeit und Sauberkeit ${ }^{3}$.

Das Stereotyp der polnischen Wirtschaft wird nach Orłowski als das sog. Stereotyp der langen Langlebigkeit klassifiziert": „Die Beschaffenheit von Stereotypen langer Dauer liegt nicht darin, dass sie lange und praktisch ununterbrochen funktionieren, sondern dass sie in jedem geeigneten Moment wieder zum Leben erweckt werden können" ${ }^{\prime \prime}$. Orłowski klärt die Zugehörigkeit der polnischen Wirtschaft zu dieser Gruppe auf die folgende Art und Weise:

Aus der Geschichte des Ausdrucks sowie der Konnotationen der polnischen Wirtschaft folgt, dass es in diesem Fall um eine Art Baumkuchen geht, in dem abwechselnd soziale, politische, geschichtsphilosophische, moralische Konzeptionen und Regeln des gesunden Menschenverstandes auftreten ${ }^{6}$.

Orłowski weist darauf hin, dass jahrelang keine empirischen Untersuchungen zum Thema das Stereotyp der polnischen Wirtschaft durchgeführt

\footnotetext{
1 Vgl. http:/ / www.kulturforum-ome.de/pdf/1000355a.pdf (14.11.2012).

2 http:/ / www.kulturforum-ome.de/pdf/1000355a.pdf (14.11.2012).

${ }^{3}$ http:/ / www.kulturforum-ome.de/pdf/1000355a.pdf (14.11.2012).

4 Vgl. http://www.kulturforum-ome.de/pdf/1000355a.pdf (14.11.2012).

${ }^{5}$ http:/ / www.kulturforum-ome.de/pdf/1000355a.pdf (14.11.2012).

${ }^{6}$ http:/ / www.kulturforum-ome.de/pdf/1000355a.pdf (14.11.2012).
} 
wurden7. Aus diesem Grund kann nicht behauptet werden, dass die erwähnte stereotype Bezeichnung der wirtschaftlichen Lage Polens spiegelt sich in der Realität wider:

Im deutschen Polendiskurs geisterte über längere Zeit ein Polenbild herum, das keine empirisch verifizierbare Entsprechung hatte, keinem nachvollziehbaren Verhältnis zwischen Zeichen, Begriff und Realzustand entsprach, ein Bild also, das mehr mit einem Phantomschmerz denn mit der Realität zu tun hatte ${ }^{8}$.

Orłowski vertritt die Ansicht, dass das negative Bild der polnischen Wirtschaft mehrmals als Selbstverständlichkeit präsentiert wurde ${ }^{9}$ :

Umso wichtiger war der funktionale Nutzen dieses schillernden Bildes von polnischer Wirtschaft, dessen sich einzelne Diskursteilnehmer nach Belieben bedienen konnten. Der deutsche Polendiskurs verfügte und verfügt wohl immer noch über eine außerordentliche moralisch-zivilisatorisch begründete Macht, sich über Polen mit Respekt bzw. despektierlich zu äußern ${ }^{10}$.

Wie bereits erwähnt, versucht die Autorin dieses Artikels festzustellen, ob das negativ assoziierte Stereotyp der polnischen Wirtschaft der imagologischen Perspektive entspricht. Aus diesem Grund wird an dieser Stelle die Klärung der imagologischen Sichtweise benötigt.

\section{ZUR KLÄRUNG DER IMAGOLOGISCHEN PERSPEKTIVE UND DER AUSWAHL DER ZUR ANALYSE STEHENDEN TEXTE}

Grob gesehen beschäftigt sich Imagologie bzw. interkulturelle Hermeneutik mit den in den Texten dargestellten Bildern anderer Länder, d.h. mit dem Versuch, die gegenseitigen Einflüsse des Fremden und des Eigenen festzustellen; mit der Bemühung, die Selbstanalyse durch Fremdanalyse (und umgekehrt) durchzuführen. Diese Ansicht vertreten z.B. die folgenden Forscher: Dyserinck (1988: 22), Mehnert (1997: 42) oder Krause (1998: 15) Laut Fischer (1987: 55) gehört Imagologie als Forschungsgebiet zu der Komparatistik: die vergleichende Literatur- und Kulturwissenschaft beschäftigt sich in ihrer historisch-vergleichenden Abteilung (d.h. zu guten Teilen im Rahmen der Beziehungsforschung) mit solchen literarischen bzw. Literatur-Phänomenen, die in mindestens in zwei Sprachgebieten als

\footnotetext{
7 Vgl. http:/ / www.kulturforum-ome.de/pdf/1000355a.pdf (14.11.2012).

8 http:/ / www.kulturforum-ome.de/pdf/1000355a.pdf (14.11.2012).

${ }^{9}$ Vgl. http:/ / www.kulturforum-ome.de/pdf/1000355a.pdf (14.11.2012).

10 http:/ / www.kulturforum-ome.de/pdf/1000355a.pdf (14.11.2012).
} 
kontaktologisch oder typologisch erklärbare Prozesse und Beziehungen identifizierbar sind (Fischer 1987: 55)

Klin beruft sich auf das wissenschaftliche Interesse am Phänomen der Modifizierbarkeit der existierenden Definitionen mithilfe imagologischer Untersuchung. Als wissenschaftstheoretischer Beitrag zu diesem Thema sind die folgenden Überlegungen gemeint: „Stereotype, die literarische Bilder darstellen, werden immer von den Autoren modifiziert" (Klin 1991: 145). Ein literarisches Werk kann also nach Klin nur durch eine genaue Analyse des Inhalts untersucht werden. Erwähnenswert bleibt die Tatsache, dass Imagologie immer öfter als interkulturelle Hermeneutik bezeichnet wird. Im Hinblick auf Gadamers (1991) Hermeneutik kann die interkulturelle Hermeneutik laut Köchler (1978: 40-43) als eine Art Basis eines „Dialoges der Zivilisationen” berücksichtigt werden und einen signifikanten Einfluss auf die zwischenkulturellen Kontakte ausüben, d.h. der Verbesserung der deutsch-polnischen Beziehungen dienen. An dieser Stelle soll der Frage nachgegangen werden, ob die in diesem Artikel analysierten Texte als eine Art meinungsbildender Vermittler zwischen dem Eigenen und dem Fremden bezeichnet werden können. Die im Fokus dieses Artikels bei interpretativer Forschung zergliederten Texte benötigen einen bestimmten Bekanntheitsgrad, der die Vermittlung der dargelegten Inhalte ermöglicht. Aus diesem Grund sollte man hier nach Pürer und Schweiger den bedeutsamen Aspekt der Medienwirkung im Hinblick auf das Schreiben berücksichtigen, der eine relevante Rolle bei der Eroberung des Publikums eines Schriftstellers spielt. Gerade auf diesem Gebiet stehen dem Verfasser eines Textes nicht nur die geschriebenen Texte in Form eines Buches zur Verfügung, sondern auch die anderen Methoden, die bei der Eroberung des Publikums Signifikanz besitzen, wie z.B.: Aussagen im Fernsehen, Radio oder Talk-Shows, Interviews in bedeutsamen Zeitungen oder Zeitschriften (vgl. Pütet 2003: 107; Schweiger 2007: 52).

In den ausgewählten Texten von Steffen Möller und Thomas Urban wird ein relevantes Bild in Erwägung gezogen - die mit der Systemveränderung und Infrastrukturentwicklung eng verbundene, sich ständig ändernde polnische Gesellschaft. Die von Möller und Thomas dargestellten Bilder scheinen zum Teil als individualistisch und gegensätzlich. Die Autoren konzentrieren sich auf die anderen Aspekte, die ihrer Meinung nach den Wirtschaftswachstum Polens zeigen. Nach der Zeit des Kommunismus kam eine ganz andere Ära, in der sowohl die Kraft der Umwandlung der polnischen Umgebung, als auch die Dynamik der Entwicklung eines neuen Modells der Gesellschaft zu berücksichtigen ist. Mit Rücksicht auf die infrastrukturelle Entwicklung und die sozialen Veränderungen in der Gesellschaft konzentrieren sich die erwähnten Schriftsteller überwiegend auf Polen, um das sog. Neue Polen zu skizzieren. 


\section{4. "VIVA POLONIA. ALS DEUTSCHER GASTARBEITER IN POLEN" VON STEFFEN MÖLLER}

Steffen Möller lebt seit 1994 in Polen. Sein Buch „Viva Polonia. Als deutscher Gastarbeiter in Polen" genießt Erfolg sowohl in Polen, als auch in Deutschland. Die Publikation beinhaltet nicht nur die den Alltag betreffenden Beobachtungen, sondern auch die unterschiedlichen geschichtlichen Aspekte, die als eine Art Hilfe zum Fremdverstehen angesehen werden können. In diesem Abschnitt soll der Frage nachgegangen werden, ob die Beobachtung Möllers Bildes der polnischen Wirtschaft mit der Rückständigkeit und Innovationsunfähigkeit assoziiert wird.

Eine erste Betrachtung verdient das Bild der Infrastrukturentwicklung konkreter polnischer Orte. Möller (2008: 340) verweist auf vier Vorzüge Warschaus. Der erste betrifft die Modernität der Stadt: „Warschau ist hervorragend geeignet zum Einkaufen, seit im Zentrum ein paar hypermoderne Einkaufs-Malls entstanden sind". Die zweite positive Eigenschaft der polnischen Hauptstadt besteht laut Möller darin, dass „in Warschau (...) bei weitem nicht so ein Verkehrschaos wie in anderen europäischen Metropolen herrscht. In Krakau ist der Verkehr schlimmer, da es dort sehr viele Einbahnstraßen gibt" (343f). Auch den Mangel an Touristen betrachtet der Essayist als Positivum: „Es gibt keine Touristen. Das ist der Vorteil der Hässlichkeit. Die Warschauer Plätze, Straßen und Einkaufszentren sind deswegen nicht überfüllt. Wer herkommt, beschränkt seine Visite meist auf die Altstadt oder verliert sich irgendwo zwischen Wilanów und Flughafen" (344). Als den vierten Vorzug Warschaus bezeichnet Möller die Möglichkeit eines Treffens mit der sog. polnischen Prominenz:

Wie in Hauptstädten üblich, treffen in Warschau alle Bevölkerungsgruppen aufeinander. In der U-Bahn sieht man Geschäftsleute neben Politikern, Fernsehleuten und Fabrikarbeitern. Weil das Zentrum aber klein ist, kann man in Warschau bei einem einzigen Bummel gleich drei Prominente sehen (344f).

Zwischen der deutschen und der polnischen Betrachtungsweise der Hauptstadt lassen sich nach Möller keine Parallelen herstellen: während die Deutschen stolz auf Berlin sind, scheint der Polen Warschau eher peinlich zu sein:

Diese merkwürdige Beziehung der Polen zu ihrer Hauptstadt könnte man als Sadomasochismus bezeichnen. In Europa dürfte er einzigartig sein. In der Regel sind die Bewohner der Hauptstädte wohl eher stolz auf ihre Stadt. Für Berliner ist Berlin die coolste Stadt Deutschlands, in einer ganz anderen Liga als Köln oder Hamburg angesiedelt (346). 
In diesem Punkt verdient die Tatsache Berücksichtigung, dass der Sadomasochismus der Warschauer auch zahlreiche positive Seiten hat:

Mir liegt der seltsame Sadomasochismus der Warschauer näher. Zugewanderte wie ich brauchen sich hier nicht wie Touristen oder Eindringlinge zu fühlen. Wir sitzen mit den echten Warschauern am Weichselufer und beneiden die Fluten, die nur wenige Stunden zuvor noch an dem Krakauer Königsschloss vorbeigeströmt sind (346).

Erwähnenswert bleibt die Tatsache, dass im postkommunistischen Polen die relevantesten Arbeitsstellen den Vertretern der jungen Generation gehören:

Polen wird - sehr im Unterschied zu Deutschland oder Japan - von der jüngeren Generation beherrscht. (...) Die wichtigsten Positionen und das große Geld sind fest in den Händen der Jungen. Dreißigjährige können in Polen schon höhere Positionen wie Abteilungsleiter oder Projektmanager bekleiden (...) (142).

Eine ähnliche Situation betrifft auch die Konsumgüter und die neu entstandenen Konsumgewohnheiten, die laut Möller auch mit der jüngeren Generation von Polen assoziiert werden (vgl. 144). Der Autor weist auf eine seiner Beobachtungen hin - der soziale Status der jungen Polen stehe im Zusammenhang mit der gesellschaftlichen Position der älteren deutschen Gesellschaft: „In Deutschland wundere ich mich inzwischen, wenn ich am Steuer eines Mercedes oder BMW einen sechzigjährigen Fahrer erblicke. Wenn man in Polen ein teures Auto sieht, kann man sicher sein, dass sein Besitzer unter fünfzig, meinst sogar unter vierzig ist" (144). Der Essayist begründet seine Beobachtung so: „Die vor 1960 geborenen Polen sind die Verlierer des Systemwechsels von 1989. Sie haben kaum eine Chance auf einen neuen Job und werden niemals Urlaub in Scharm el Scheik machen. Dort laufen junge Polen alten Deutschen über den Weg" (144).

Möller betrachtet Polen am Arbeitsplatz als eine arbeitsame und fleißige Nation:

Schrankenlos bewundern kann ich nur das Verhältnis der Polen zur Arbeit. Obwohl die Wirtschaft boomt und Arbeitszeiten bis zu siebzig Wochenstunden durchaus üblich sind - nicht umsonst gilt Warschau als die Stadt mit der längsten Arbeitszeit in Europa - wird der Arbeitsplatz immer noch nicht mit einem Glorienschein umgeben, so wie in Deutschland (34f).

Der Autor erwähnt auch einen relevanten Grund dafür, warum die Deutschen aus ihrer Perspektive die polnischen Angestellten als inkompetent in Erwägung ziehen können. Es handelt sich vor allem um Herzlichkeit, Optimismus und Fröhlichkeit am Arbeitsplatz: 
Wie ungewohnt war es für mich in Polen, als ich bei der Kontoeröffnung im Hintergrund der Bank fröhliches Lachen hörte. Wie perplex war ich, als der Klempner plötzlich über seine Familie sprach. Meine spontane Befürchtung war sofort: Wer solche Scherzchen während der Arbeit macht, kann nicht viel Kompetenz besitzen (35).

Laut Möller ist das Wort Rückständigkeit kein gutes Synonym für polnische Wirtschaft. Dies bestätigt auch die Untersuchung des Wirtschaftswachstums Polens - die Veränderung des realen BIP im Jahr 2009 glich ca. 1,6\%, und im Jahr 2011 - ca. 4,5\%11. Das ganze Land erinnert zurzeit an eine riesengroße Baustelle: es wird ständig renoviert oder gebaut. Wegen der ununterbrochenen Bauarbeiten stehen die Bewohner der polnischen Großstädte täglich - und ab und zu stundenlang - im Stau. Der Publizist vertritt die Meinung, dass an dieser Stelle nur ein Aspekt der polnischen Gesellschaft manchmal stören kann - die Planlosigkeit im Bauwesen im Hinblick auf das fehlende Gefühl der Schönheit (103):

Was mich an der polnischen Freiheit rasend macht, ist die unglaubliche Planlosigkeit, mit der das Land kreuz und quer verbaut wird. Lasche, korrupte Bauaufsichtsbehörden lassen chaotische Siedlungen entstehen, wo jeder sein Dach hellblau und die Garage dunkellila anmalen kann; es sieht fürchterlich aus (103).

Zusammenfassend lässt sich feststellen, dass Möller auf verschiedenartige Entwicklungen Polens verweist. Die erwähnten Veränderungen betreffen die unterschiedlichen Lebenssphären. Möllers Ansicht nach sollte Stereotyp der polnischen Wirtschaft nicht mehr als Synonym der wirklichen gegenwärtigen Situation in Betracht genommen werden.

\section{5. „POLEN" VON THOMAS URBAN}

Der aus Breslau stammende deutscher Osteuropa-Korrespondent der "Süddeutschen Zeitung" und Publizist Thomas Urban beschäftigt sich seit langem mit den deutsch-polnischen Beziehungen, die die verschiedensten Aspekte betreffen: die Vertreibung der Polen und der Deutschen, das Leben der Deutschen in Polen, usw. Urban hat zwei bedeutsame Auszeichnungen bekommen: den Georg Dehio-Buchpreis im Jahr 2006 für die Publikation "Verlust. Die Vertreibung der Deutschen und Polen im 20. Jahrhundert” und die Bayerische Europamedaille im Jahr 2010.

\footnotetext{
${ }^{11}$ http://wko.at/statistik/eu/europa-wirtschaftswachstum.pdf (15.08.2013).
} 
Die Autorin dieses Artikels konzentriert sich auf die Veröffentlichung von Urban unter dem Titel „Polen”. Der Schriftsteller beschreibt die gegenwärtige Situation Polens aus den unterschiedlichen Perspektiven: Geschichte, Politik und Wirtschaft, Gesellschaft und Kultur.

Das mit der Rückständigkeit und mangelhafter Entwicklung verbundene Stereotyp der polnischen Wirtschaft entspricht nach Urban (2008: 123) der jetzigen polnischen wirtschaftlichen Lage nicht mehr - Polen wird hier mit dem Wirtschaftsboom konnotiert. Der Autor beruft sich an dieser Stelle auf den polnischen Wirtschaftswissenschaftlern und ehemaligen Vizepremier und Finanzminister, Leszek Balcerowicz. Der Balcerowicz-Plan steht in Verbindung mit der Umstellung der Zentralplanwirtschaft Polens auf die Marktwirtschaft:

Der Balcerowicz-Plan sah die Schaffung eines Finanz- und Bankensystems nach westlichem Vorbild vor. Auf diese Weise wurde Kapital für Investitionsvorhaben aller Art bereitgestellt. Dass Kapital vorhanden war, machte sich zunehmend im Straßenbild bemerkbar: Häuserfassaden wurden renoviert, Geschäfte modernisiert, in allen Großstädten brach ein Bauboom aus (123).

Urban bezeichnet die polnische Gesellschaft als Konsumgesellschaft, die zahlreiche Sphären des Lebens beeinflusst: „Die polnischen Konsumenten können sich an das neue Gefühl gewöhnen, in Geschäften, Supermärkten und Warenhäusern einkaufen zu gehen, die kaum den westlichen Vorbildern nachstehen" (124). Der Journalist erwähnt auch die Änderung der Einstellung den Kunden gegenüber:

Ebenso machte das Heer der Verkäufer und Verkäuferinnen eine Metamorphose durch: Jahrzehntelang in der Defizitwirtschaft daran gewöhnt, dass der Verkäufer König war, schaffte es die Mehrheit schnell, sich darauf einzustellen, dass nun der Kunde König ist. Überdies explodierte die in der Planwirtschaft völlig vernachlässigte Dienstleistungsbranche förmlich (124).

Polen als Mitglied der Europäischen Union gleicht den anderen, zu der EU gehörenden Staaten auf unterschiedliche Art und Weise, die die bestimmten Sphären des Lebens und den entsprechenden Lebensstil betrifft. Ein gutes Beispiel dafür scheint der Automobilmarkt in Polen zu sein: „Während Anfang der 90er Jahre das Straßenbild von Polski Fiat, Polonez, Skoda, Wolga, Wartburg und Trabant bestimmt wurde, hatten ein halbes Jahrzehnt später deutsche, italienische, französische, japanische und koreanische Anbieter einen Großteil des Markts übernommen" (124). Dies wird von Urban auf die folgende Art und Weise geklärt: 
Da Polen als der wichtigste Wachstumsmarkt für Automobile in Europa galt, die Regierung gleichzeitig aber die eigene Automobilindustrie zunächst durch hohe Importzölle schützte, bauten mehrere Konzerne eigene Werke in Polen, darunter Opel, Volkswagen, Peugeot, Fiat und Mercedes für Nutzfahrzeuge (...). In Warschau beispielsweise hat sich die Zahl der Personenwagen zwischen 1990 und 2000 verzwölffacht (...) (124).

Mittlerweile wird laut Urban das neue EU-Mitglied Polen nicht mehr als eine Nation "des Rückstands", sondern als ein geeignetes Land für unterschiedliche Investitionen aller Art angesehen. Selbstverständlich betrifft dies nicht nur den oben erwähnten Automobilmarkt, sondern auch andere Bereiche der Wirtschaft:

Auch andere Industriezweige verzeichneten zunächst riesige Produktionszuwächse. So lassen deutsche, niederländische und französische Weltfirmen in Polen Haushaltsgeräte bauen. Internationale Lebensmittelkonzerne produzieren in Polen für den gesamten osteuropäischen Markt, aber auch für den Export nach Westeuropa (124f).

Die Mitgliedschaft Polens in der Europäischen Union ist nach Urban eng mit der Entwicklung der Agrokultur verbunden:

Die Umstrukturierung der Landwirtschaft stellte eine der größten Herausforderungen für die polnische Politik auf dem Weg in die EU dar. In der Tat machte auch der Agrarsektor einen Modernisierungsschub durch. Die Lebensmittelindustrie erfüllte rasch die EU Normen. Gleichzeitig wurde die Produktion der Agrargüter weitgehend mechanisiert (127f).

Urban vertritt die Ansicht, das das bekannte Stereotyp der polnischen Wirtschaft nicht mehr existiert: „Das Bäuerlein hinter dem von einem Pferd gezogenen Pflug - damit illustrieren westliche Medien gern den Zustand der polnischen Wirtschaft - wurde innerhalb weniger Jahre zur aussterbenden Species". Der polnische Wirtschaftsboom hinterlässt nach Urban auch kulturelle Spuren - es sind unterschiedliche Orientierungen vorhanden (177):

Auch die polnische Gesellschaft orientiert sich zunehmend an den Werten und Ausdrucksformen der westlichen Nachbarn, wie der Deutschen und Franzosen, teilweise auch der Amerikaner. (...) Zweifellos geht von den USA ein starker, kultureller Einfluss aus, den die Kirche und konservative Gruppierungen mit großer Sorge betrachten (177).

Der Autor erwähnt hier z.B. die Amerikanisierung der jungen polnischen Generation, die das reiche Spektrum der unterschiedlichen Aspekte 
betrifft, die Durchsetzung der amerikanischen Vokabeln in der Jugendsprache, die mit der Gewalt auf dem Bildschirm eng verbundene Zunahme der Gewaltverbrechen, die auf den Musiksender MTV und Markenartikel konzentrierte Jugend, usw. (177).

\section{ZUSAMMENFASSUNG}

Zusammenfassend kann man zu dem Schluss kommen, dass die dargestellten Bilder in den zur Analyse ausgewählten Texten dem bisherigen, mit der Rückständigkeit und fehlenden Innovationsfähigkeit assoziierten Stereotyp der polnischen Wirtschaft nicht entsprechen. Nach dem EU-Beitritt sind in Polen unterschiedliche Veränderungen eingetreten. Sie betreffen zahlreiche Lebenssphären: sowohl die privaten Angelegenheiten, als auch die wirtschaftliche Lage. Laut Steffen Möller und Thomas Urban wird Polen heutzutage mit den verschiedenartigen Entwicklungen konnotiert. Die Gesellschaft wird immer reicher - die heutigen Polen sind Teil einer modernen Konsumgesellschaft: Sie sind imstande, eine exotische Auslandsreise, einen exklusiven Mercedes oder andere Luxusgüter zu erwerben. In den letzten Jahren erinnert das ganze Polen an eine riesengroße Baustelle - es wird ununterbrochen und fast überall renoviert und gebaut. Auch unterschiedliche Industriezweige, wie z.B. Automobilindustrie oder Landwirtschaft, unterliegen Modifizierungen. Andererseits existiert immer noch das Stereotyp der polnischen Wirtschaft, das als synonyme Bezeichnung für wirtschaftliche Rückständigkeit und mangelhafte Entwicklung in Erwägung gezogen wird. Solche Veröffentlichungen, wie Möllers „Viva Polonia. Als deutscher Gastarbeiter in Polen" oder Urbans "Polen”, beschreiben die aktuellen Bilder der gesellschaftlichen Lage Polens, die mit der bisher überwiegend auf eine negative Art und Weise assoziierten polnischen Wirtschaft in keinem Zusammenhang stehen. An dieser Stelle entsteht die Frage, ob die hier analysierten Publikationen imstande sind, das bestehende Stereotyp zu modifizieren? Weil Stereotype als generalisierende und stark im Gedächtnis geprägte Meinungen verstanden werden, wird dies als keine einfache Aufgabe angesehen. Die Antwort auf die oben gestellte Frage kann also noch nicht gegeben werden, weil Stereotype zwar modifizierbar sind, aber der Prozess ihrer Modifikation sehr langsam verläuft. Es bleibt die berechtigte Hoffnung, dass die polnischen Wirtschaft und Gesellschaft im Laufe der Zeit als ein Synonym für Modernisierung und Innovation angesehen werden. Die meinungsbildende Rolle der Medien (z.B. Veröffentlichungen aller Art, Fernsehen, Internet oder Rundfunk) wird dabei behilflich sein. 


\section{LITERATURVERZEICHNIS}

Adler, N.J., Gundersen, A., 2008. International dimensions of organizational behavior, Fifth Edition. Mason, $\mathrm{OH}$ : Thomson South-Western.

Auderska, H., Łempicka, Z., Skorupka, S., 1968. Mały słownik języka polskiego. Warszawa: Wydawnictwo Naukowe PWN.

Auerheimer, G., 1990. Einführung in die interkulturelle Erziehung. Darmstadt: Wissenschaftliche Buchgesellschaft.

Chlebda, W., 1998. Stereotyp jako jedność języka, myślenia i działania. In: Anusiewicz, J., Bartmiński, J. (Hrsg.) Jezyk a kultura, tom 12: Stereotyp jako przedmiot lingwistyki. Teoria, metodologia, analizy empiryczne. Wrocław: Towarzystwo Przyjaciół Polonistyki Wrocławskiej, 31-40.

Dyserinck, H., 1988. Komparatistische Imagologie. Zur politischen Tragweite einer europäischen Wissenschaft von der Literatur. In: Dyserinck, H., Syndram, K.U. (Hrsg.) Europa und das nationale Selbstverständnis. Imagologische Probleme in Literatur, Kunst und Kultur des 19. Und 20. Jahrhunderts. Bonn: Bouvier, 13-37.

Fiedor, K., 1997. Wokół stereotypu „polnische Wirtschaft”. In: Zbliżenia 3 (18), 92-95.

Fischer, M.S., 1987. Literarische Imagologie am Scheideweg. Die Erforschung des "Bildes vom anderen Land" in der Literatur-Komparatistik. In: Blaicher, G. (Hrsg.) Erstarrtes Denken. Studien zu Klischee, Stereotyp und Vorurteil in englischsprachiger Literatur. Tübingen: Gunter Narr Verlag, 55-71.

Fiske, S.T., Operario, D., 2004. Stereotypes: Content, Structures, Processes and Context. In: Brewer, M.B., Hewstone, M. (Hrsg.) Social Cognition. Malden/MA: Blackwell Publishing Ltd, 120-141.

Gadamer, H.-G., 1990. Wahrheit und Methode. Band 1. 6. Auflage. Tübingen: MohrSiebeck Verlag

Golec, J., 2000. „Polnische Wirtschaft” - potęga stereotypu. In: Borussia 20/21, 305308.

Hansen, K.P., 2000. Kultur und Kulturwissenschaft. Eine Einführung. Tübingen und Basel: A. Francke Verlag.

Hogg, M., Vaughan, G., 2010. Essentials of Social Psychology. Harlow: Pearson Education.

Klin, E., 1991. Stereotypy etniczne w literaturze. In: Wrzesiński, W. (Hrsg.) Wokót stereotypów Polaków i Niemców. Über die Stereotype der Polen und der Deutschen. Wrocław: Acta Universitatis Wratislaviensis 1136, 143-151.

Köchler, H., 1978. Kulturphilosophische Aspekte internationaler Kooperation. In: Zeitschrift für Kultur-Austausch, Bd. 28, 40-43.

Krause, T., 1998. Die Fremde rast durchs Gehirn, das Nichts... Deutschlandbilder der Banater Autorengruppe (1969-1991). Studien zur Reiseliteratur- und Imagologieforschung 3. Frankfurt am Main: Lang.

Lippmann, W., 1922/2008. Public Opinion. New York: Harcourt, Brace and Company.

Mehnert, E., 1997. Bilderwelten - Weltbilder. Vademekum der Imagologie. Chemnitz.

Möller, S., 2008. Viva Polonia. Als deutscher Gastarbeiter in Polen. Frankfurt am Main: Scherz. 
Orłowski, H. "Polnische Wirtschaft". Ausformung eines hartnäckigen Vorurteils. http:/ / www.kulturforum-ome.de/pdf/1000355a.pdf (14.11.2012).

Orłowski, H., 2004. Die Lesbarkeit von Stereotypem. Der deutsche Polendiskurs im Blick historischer Stereotypenforschung und historischer Semantik. Wrocław: Wrocławskie Wydawnictwo Oświatowe.

Papiór, J., 1997. Diskurs zum Stereotyp der „polnischen Wirtschaft”. In: Zbliżenia $3(18), 95-98$.

Pürer, H., 2003. Publizistik- und Kommunikationswissenschaft. Ein Handbuch. Konstanz: UVK Verlagsgesellschaft $\mathrm{mbH}$.

Schneider, D.J., 1996. Modern Stereotype Research: Unfinished Business. In: Hewstone, M. Macrae, C.N., Stangor, C. (Hrsg.) Stereotypes and stereotyping. New York: The Guilford Press: 419-454.

Schweiger, W., 2007. Theorien der Mediennutzung. Eine Einführung. Wiesbaden: VS Verlag für Sozialwissenschaften-GWV Fachverlage GmbH.

Stangor, C., 2000. Stereotypes and prejudice. Philadelphia: PSYCHOLOGY PRESS Taylor and Francis Group.

Urban, T., 2003. Polen. 2. Auflage. München: Verlag C.H. Beck oHG.

http:/ / wko.at/statistik/eu/europa-wirtschaftswachstum.pdf (15.08.2013). 